\title{
B4HEALTH - AN ARCHITECTURE MODEL FOR PERSONAL HEALTH RECORDS WITH HL7 FHIR AND HYPERLEDGER FABRIC
}

\author{
Victor Giovanella Garcia, Alex Roehrs, Cristiano André da Costa, \\ Rodrigo da Rosa Righi, André Henrique Mayer, Rodolfo Stoffel Antunes \\ and Eduardo Souza dos Reis \\ Unisinos Software Innovation Laboratory - SOFTWARELAB / Universidade do Vale do Rio do Sinos - UNISINOS \\ Av. Unisinos, 950 - Bairro Cristo Rei - São Leopoldo/RS CEP: 93.022-750, Brazil
}

\begin{abstract}
Health Institutions use the EHR (Electronic Health Record) for many years to register the patients' information electronically. On the other hand, this data is still in many different systems of the health institutions, which the patient interacted over life. During a medical appointment, one does not have a current view of the patient's health history. One of the goals of the PHR (Personal Health Record) concept is to obtain a unified view of the records scattered in various health organizations. Many experts now believe that blockchain technology allows storing a patient's pertinent medical information where needed, allowing both the patient and health care professionals to have access to up-to-date information. However, among the challenges to achieve the goal of an interoperable PHR model is the choice of a blockchain platform that supports the storage of this data in a secure, transparent, and decentralized manner. This article proposes an architecture model for health records based on blockchain. The main outcome is storing part of an electronic medical record in the proposed solution. It aims to process and store medical data, which comes from different health institutions safely inside the blockchain network. Then the EHR data is available to authorized stakeholders in a unique viewpoint through a decentralized blockchain application. After experimenting with two different blockchain frameworks, we decided to implement the prototype in a permissioned blockchain. We created and deployed a business network composed of two different organizations, which stored and shared EHR data among the network participants. Performance tests while retrieving EHR data from the blockchain showed an average response time in milliseconds of 201.08 with 500 users and $15,402.9$ with 1000 users. Our proposed solution was able to address the main challenge faced by patients that have EHR data scattered in many distinct locations. The prototype supplies a unique view of this data where the participating organizations accessed the EHR data. However, a more robust hardware setup is necessary to test the blockchain solution in future works. Additionally, our intent is to focus on internal aspects of HIS, especially on how to convert the current EHR data from any health data standard to HL7 FHIR, as this would allow many different health institutions, which are using different data standards to take part in the network.
\end{abstract}

\section{KEYWORDS}

HER, PHR, Blockchain, Healthcare, HL7 FHIR

\section{INTRODUCTION}

According to Pons et al. (2016), the increase in Electronic Health Records (EHR) usage is generating new challenges and opportunities in the medical field, and increasing the use of digital content, both structured data as well as narrative text should offer many benefits. In addition to the original goal of improving clinical care through high quality documentation, EHR data can make contributions that are more significant to research and clinical workflows. In the health sector, although we live in the information age and there are already several systems that make the EHR of patients, the information is still spread in several systems of different institutions such as hospitals, ambulatories, clinics and doctors' offices. Authors in Fan et al (2018), believe the problem is that EHRs stay in a private database making patients leaving their data in various hospitals, this usually happens because life events take them from one hospital to another. Therefore, most patients lose access to past data even when it belongs to them. Moreover, according to Zhang et al (2018), it is essential to have 
secure and scalable data sharing for patients to receive effective treatment. During their lifetime, patients visit a variety of care provider's offices and to ensure these care providers have the most up to date information about their patients' health condition, they should be able to exchange information in timely and privacy-sensitive manner.

To solve the problem of information spread in different places, the author Roehrs et al proposed a Personal Health Record (PHR) model that aims to unify data from several sources. This model aims to provide patients with a unified view of their health records scattered in various locations. It also aims to make available to health professionals' access to updated patient data, even when changed in other health institutions with which the patient interacted. The OmniPHR model focuses on the distribution and interoperability of the PHR. The OmniPHR proposes a representation of the PHRs, organized hierarchically, encrypted, and distributed in chain databases in the network. These blocks can be in different healthcare organizations and even in a patient-managed repository. As OmniPHR's proposal is to promote an interoperable and distributed PHR architecture with security features for sensitive data, the authors found in the blockchain technology proper alternatives to compose the model's base architecture (ROEHRS et al, 2019).

There are some challenges faced by the OmniPHR model, such as the choice of the blockchain platform that will compose the model. Currently there are several blockchain platforms available in the market, among the main ones are Ethereum, Hyperledger Fabric, R3 String, Ripple and Quorum. Despite the popularization of the blockchain through Bitcoin, the behavior of a system that stores health data on a blockchain platform is not yet well known. It is unknown, which of the major platforms offer the best features to support the OmniPHR model, for the storage of health data. This work aims at creating a blockchain architecture model to store EHR data using a health data standard to support the OmniPHR model.

\subsection{Scientific Contribution}

There is a growing need for an interoperable health data system that supplies a common shared view of the essential data for a person and any healthcare professional involved in care, regardless of where the provider or the person is physically located, or the organization to which they belong. Too often, the people involved in the health process waste time by repeating information gathering, besides they lose critical information, or the patient provides it too late (AZARMA et al, 2017). The EHR may have scanned patient data, however getting this data from one clinician to another is still a major challenge, especially since patients often have multiple doctors requesting tests, prescribing medications, and supplying treatment. Many experts now believe that blockchain technology allows to store a patient's pertinent medical information where needed, as well as allowing patients to easily view their own medical histories. In addition, blockchain technology can also help with other aspects of health care, such as improving the application for insurance or other administrative processes within health networks, and health-related population data available to biomedical researchers (MERTZ, 2018).

This study aims to address the issues surrounding the creation of a blockchain-based health records architecture model. The research developed a blockchain model for the storage of a partial electronic medical record using the HL7 FHIR (Health Level Seven - Fast Healthcare Interoperability Resources) standard in Hyperledger Fabric. Besides the implementation the authors also executed some performance tests.

\subsection{Research Question}

The use of blockchain has an interesting potential for the storage of EHR because it offers a secure and distributed database that can run without a central authority or administrator. The blockchain has also gained interest as a platform to improve the authenticity and transparency of health data through many use cases, such as keeping permissions on EHR to simplify the processing of requests (ANGRAAL et al, 2017). For Dubovitskaya et al (2018), nowadays it is still a major challenge on keeping a patient's medical history with up to date information, because patients usually interact with many health organizations. Blockchain provides a unique opportunity to create secure and trustable health data management solution, for sharing important patient data between healthcare providers and researchers.

Although the use of the OmniPHR model can solve some of the problems faced by health organizations and their patients, it would still be necessary to choose a blockchain platform that best supports the implementation of the model. The currently available blockchain platforms are generic, being able to store any 
type of data, there is no specific platform for health data. With few studies involving the main available platforms, it is currently unclear which one offers the best features when it comes to storing health records that use health data standards such as HL7 FHIR. The B4Health is an implementation inspired in the OmniPHR model which aims at solving the challenges mentioned above.

\subsection{Motivation}

Health organizations implemented the EHR and it is routinely in use in most European countries. In addition to the use in patient care, these data have secondary use in analyzes, research, quality measurements, among others (RIKKA VUOKKO et al, 2017). As per Reisman (2017) interoperability itself is complex. The term refers to more than just the ability to exchange information. For two EHR systems to be truly interoperable, they must be able to exchange and use this information exchanged. For this to occur, the transmitted message must have standard coded data so that the receiving system can interpret it. However, the lack of standardized data is an issue that has plagued the United States health system for decades and now certainly limits the ability to electronically share patient data. For Zhang et al. (2018), to overcome such situation, it is necessary to have a standards-based architecture, which can integrate with existing HIS and related mobile applications to enable safe and scalable systems to improve decision support.

According to Mettler (2016), a constantly updated decentralized database has many advantages for the healthcare industry. These advantages become especially interesting when many different parties need to access the same information. Medical treatment processes, for example in care for the elderly or chronic diseases, are predestined fields of application, where Blockchain technology can create benefit. A variety of stakeholders (for example, general practitioners, specialist physicians, hospitals, therapists, etc.) and the amount of disruption in the media that disseminates the information involved during the treatment of a patient (e.g. change of media, multiple medical records, incompatible IT interfaces, etc.) can lead to lengthy, resource-intensive authentication and information processes for all medical stakeholders. Considering the potential of the blockchain for health data storage in a transparent, decentralized, and secure way, this project proposes the creation of a blockchain architecture model to store EHR, discovering its advantages and disadvantages, simulating the behavior of a real scenario.

\subsection{Objective}

The general goal is to develop a blockchain architecture model for storing part of an electronic medical record. For this, there are some specific goals, such as use the HL7 FHIR as the data standard for storing information on the blockchain platform. Store information about a patient's personal data that is on an electronic medical record that is using the HL7 FHIR standard. Compare the performance of the blockchain architecture model created with two other blockchain platforms, the consumption of hardware resources, analyze response time, and verify which platform had the least errors during the tests. Determine if the result obtained gives indications that these platforms would support the storage of data using the HL7 FHIR standard in a real scenario.

\section{B4HEALTH}

According to the main problem presented in this study, the EHR data needs to sit in a central repository ensuring both the security and confidentiality. To address this issue raised by the authors of the OmniPHR model, we proposed a blockchain architecture model for EHR storage called B4Health (Blockchain for Health). B4Health aims to solve the problem of scattered EHR data in many different systems of the health institutions with which the patient interacted throughout life. The authors also created it with the goal to serve as a blockchain model to support the OmniPHR as a repository to store the EHR data. Several health institutions, such as hospitals, clinics, doctor's offices, generate EHR data and feed the B4Health system with the information generated after each appointment. In B4Health, only healthcare professionals belonging to a health institution member of the network have access to patient data. The B4Health architecture is composed of several components, this section explains them in detail. In Figure 1, it is possible to have an overview of the system, where the different health institutions send the data safely, after it arrives in B4Health and the system treats and stores the EHR data. In the last step, the system enables both patients and health professionals, who have access to the shared EHR data to use it for helping in the patient treatment. 


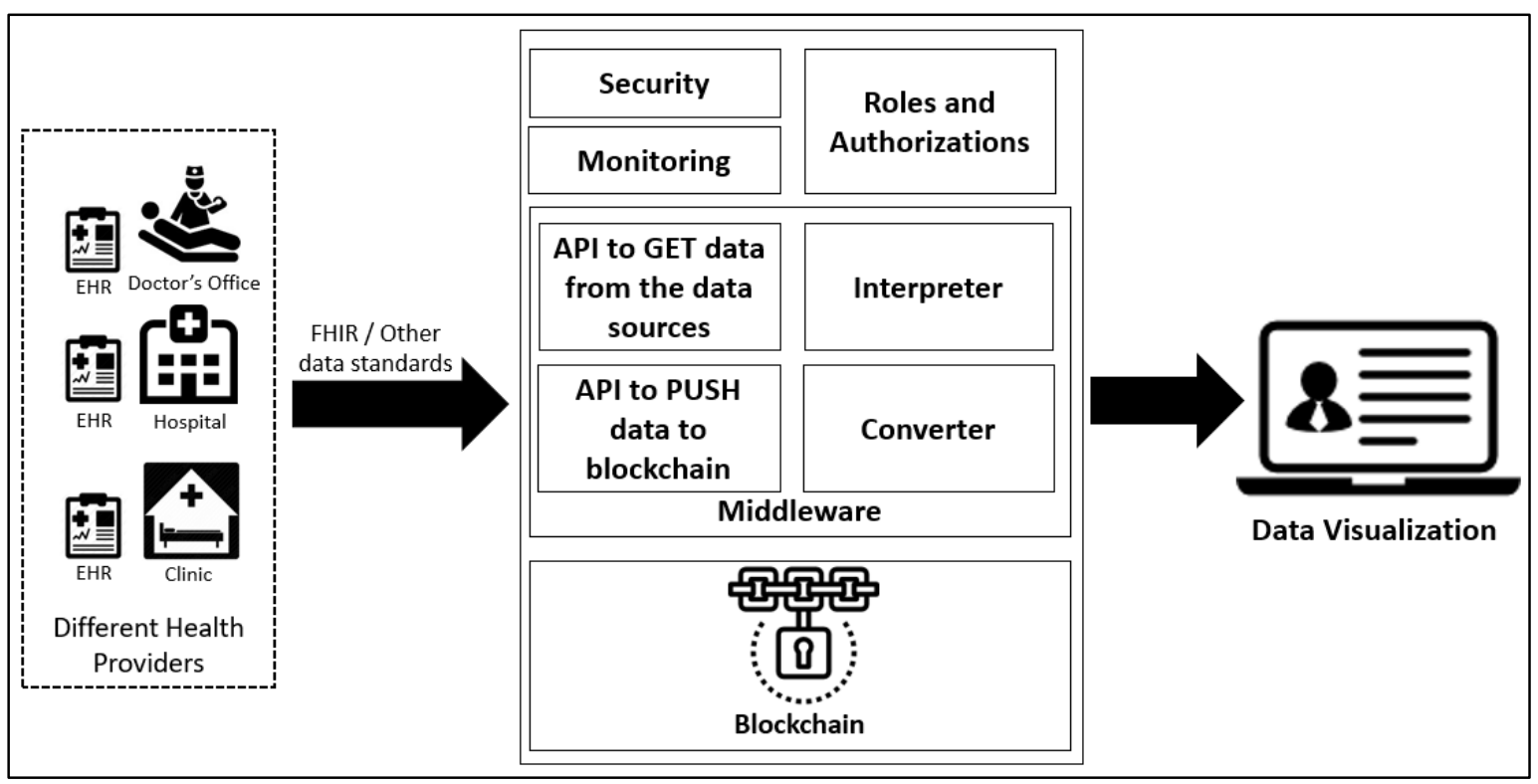

Figure 1. B4Health System Architecture

According to Roehrs et al (2017), safety is a key factor when dealing with health data. The security component is responsible for ensuring that the data leaves health care institutions and goes to B4Health, ensuring the confidentiality, privacy and integrity of the EHR. For this to be possible, we used encryption, digital certificates, and a mechanism for authentication of users who want to gain access to the information. Only health professionals authorized by the patient can access the data. For this, the authors created a structure with user roles. Each of these users has specific authorizations that differ them from one another. We created multiple roles with authorization objects tied to them. The patient can customize them to supply access to health professionals as needed and is able to fully control the access to their information available in B4Health. The monitoring component is for the user to monitor who has access to their data. Through this component, one knows who has permission to access the data. The patient can review a list of people who have access to the EHR data and decide whether to maintain or revoke the institutions' access to shared EHR data. The Data Management API handles fetching the data in the institutions' systems and stores it in B4Health. It authenticates with a user provided by the health institution to fetch the data through an exposed service.

The system encrypts the data before sending over the network or a VPN tunnel can be set up to ensure security between the senders and target system. The data converter is a key component when the system is dealing with data that does not follow the FHIR standard. The main role of the converter is to "translate" the EHR data that is using a different data standard (open or proprietary). This translation occurs automatically or with minimal user interaction. The converter is always looking to preserve the richness of the data. As many authors support the blockchain technology as a key solution for the future of EHR data sharing. We decided to experiment with the most known blockchains frameworks nowadays, Ethereum and Hyperledger Fabric. After exploring both frameworks, the authors started the prototype implementation using Hyperledger Fabric. The reasons that supported this decision are available in the Evaluation and Discussion section of this work. The patient and health professionals can access the EHR after the authentication with user/password and certificates occur. The health professional can access the patient data to which he or she has permission.

\subsection{Results}

The implementation started with the development environment provision, two virtual machines (VM) were setup with the operating system Ubuntu. The VMs had 2 CPUs Intel(R) Core (TM) i5-7300U CPU @ 2.60GHz and 4 GB of RAM each. The authors installed all requirements and dependencies along with the blockchain frameworks in each VM. As the next step, we started experimenting in each environment with two different blockchain frameworks, Ethereum and Hyperledger Fabric respectively. During this exploration phase, the goal was to become familiar with both frameworks and create a blockchain application capable of dealing with 
data from the ledger. The authors chose to start the prototype implementation in the Hyperledger Fabric framework, and to accelerate the development we used Hyperledger Composer, which is an open development toolset and framework that makes developing Hyperledger Fabric blockchain applications easier. A scenario of two hospitals exchanging patient EHR information in a private blockchain network was setup. To carry out such scenario, the blockchain network was comprised of two different organizations; each one had two peers, which were responsible to host the ledgers and chaincode. There was one certificate authority (CA) per organization in charge of distributing certificates to the involved actors. We deployed these components in one single host and implemented them as containers in a Docker runtime environment. After the blockchain network was setup, we modeled the B4Health business network and deployed it to the blockchain network.

The authors considered many aspects for the model evaluation. We started by researching and experimenting with different blockchain frameworks, then after many weeks of research and investigation we deployed the application to the chosen framework, deciding to implement the solution in a private blockchain. We used Apache JMeter to generate load against the Hyperledger Fabric blockchain network by simulating a real use case scenario where hundreds of users were accessing the application to retrieve patient EHR data that was already in the blockchain database. We manually loaded the EHR data to the blockchain prior to the tests. The authors configured Apache JMeter to send GET requests to the REST Server to retrieve information from the blockchain. Since the EHR data retrieval is the most common operation performed by the blockchain network participants, we collected and evaluated the performance of such operation. Each GET request retrieved 100 EHRs in text format, each received message body size was around 54557 bytes, while the number of sent bytes from JMeter was 129 . JMeter generated load threads with a variety of users ranging from 50 to 1000. We used the following formula for data collection.

$$
\text { Average Latency time }=\frac{S U M \text { of latency time per GET request }}{\text { Total number of GET requests }}
$$

After we deployed the business network in the blockchain network, we tested it through a simulation of a real use case scenario. As there were two different organizations (Organization 1 and Organization 2), the examination began by adding members to the different organizations, these members received certificates to be able to access the business network through a REST server and perform actions with which they had permissions. Only authenticated members of the network could perform any EHR data manipulation. Considering the proposed scenario of this work, which was to create an application for patients to have a central point of access to their EHR information, in a real-life scenario the most common operations are data creation and data retrieval. For the prototype creation, the authors modeled partially the EHR asset with the Clinical Impression resource from HL7 FHIR standard, this resource is the outcome of the clinical assessment process.

Even though the prototype was running in a virtual machine with a few resources, the authors wanted to perform a stress test to understand the behavior under heavy load. We configured JMeter with 1500 users that executed GET requests for a period of 2 minutes. After the test began, the network stopped responding after 24 seconds, during which JMeter added around 12 users per second. While the network was still responding, the average response time per request was of 21,543.08 ms (milliseconds). When all the VM CPU resources were completely exhausted, the peers running in the Docker container environment stopped responding and went down. 


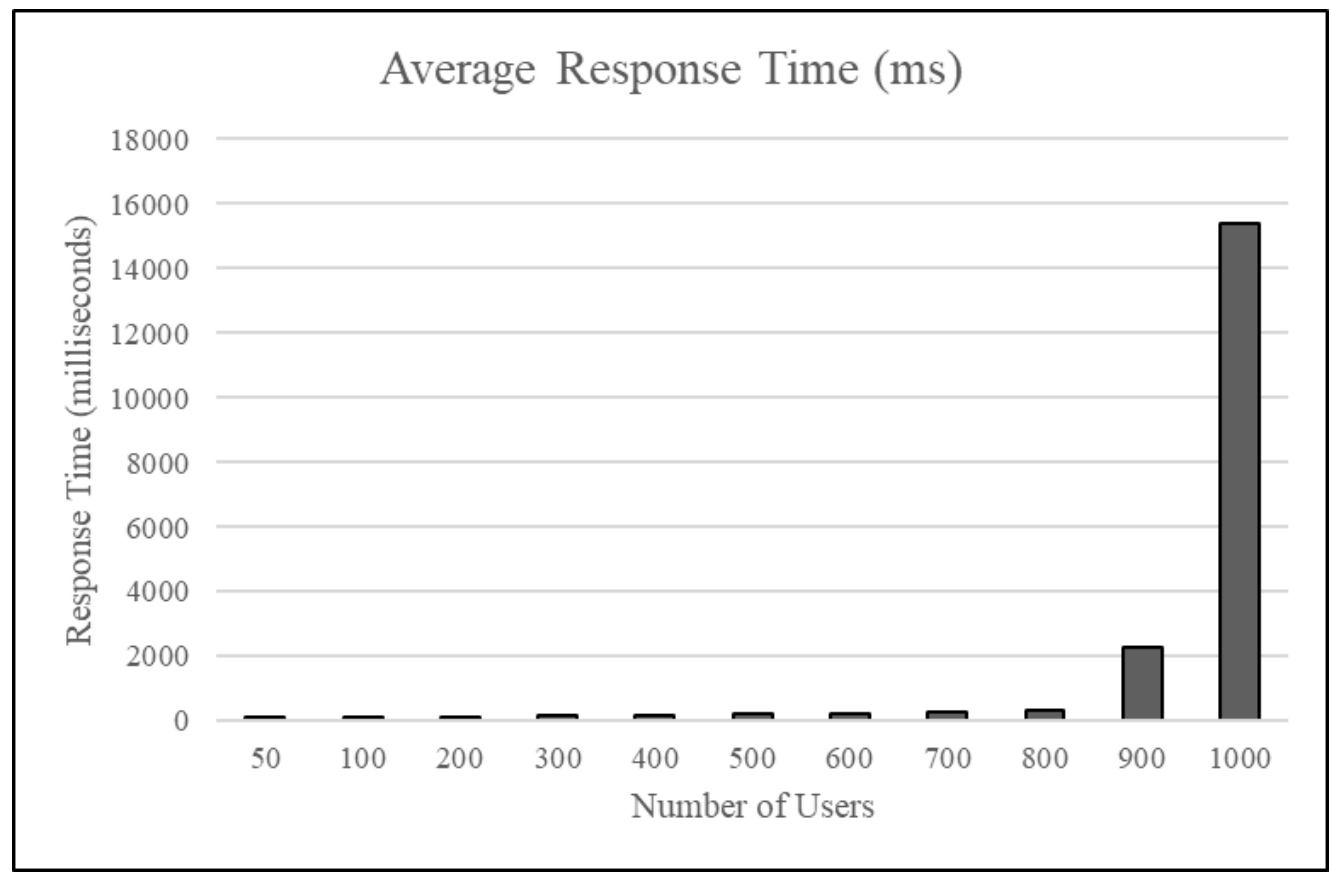

Figure 2. Average Response Time in Milliseconds

\subsection{Discussion}

Researches from around the world are working in many different projects regarding blockchain in the healthcare sector. For example, recently Chen et al (2019) proposed a blockchain-based encryption scheme for EHRs for different medical organizations and participating individuals (e.g. doctors, hospitals, medical labs, and insurance companies) to access EHRs stored in the blockchain with a higher level of trust. The authors found in the blockchain technology potential to share medical data safely, since EHR data leaking may result in compromising patient privacy. Alonso et al (2019) conducted a review of the existing research works in the literature, which conclude that the blockchain technology applied in eHealth finds new ways of sharing the distributed view of health data and promotes the advancement of precision medicine, improving health and preventing disease. His review of the research works shows that $5.56 \%$ of these works correspond to the year $2016,22.22 \%$ to 2017 and $72.22 \%$ to 2018 , which clearly tells that the research field interest on blockchain for healthcare is increasing over the past years.

After spending a couple of weeks experimenting with a simple setup of Ethereum and Hyperledger Fabric, we chose to continue with the prototype implementation using Hyperledger Fabric. The authors wanted to avoid the expenses related to Ethereum which has its own cryptocurrency, making mining a necessary requirement to run applications productively. On the other hand, Hyperledger Fabric is a private blockchain which does not require mining, it preserves the anonymity of users, making it impossible to verify the identity of account holders, and additionally it is an organization supported by more than 250 companies, including renowned key players from the global health sector industry.

Before starting the development of the blockchain application itself, we had to setup the blockchain network. The authors did not carry out the Hyperledger Fabric network setup easily, they had to learn about the infrastructure aspects of the blockchain network and its components. Fortunately, the Hyperledger Fabric has a wide documentation around the framework and an active online community to support its users. Most of the issues found during the infrastructure setup were about the installation and the network startup. Although the infrastructure setup was challenging, the blockchain application development using Hyperledger Composer framework helped to speed up the development related activities. It allowed to rapidly develop the B4Health use case and deployed the blockchain solution to the blockchain network. The authors chose the tests presented before because of the similarities with typical activities from daily operations executed in health institutions, but they executed them considering the specific use case scenario covered by this work. For example, when 
someone goes to a medical appointment, usually the doctor asks the person to describe the health history. However, in a scenario where there is a unique viewpoint of patient's EHR data, the doctor would retrieve the EHR history from the system, therefore there would be a lot of parallel reading requests happening. Similarly, after the medical appointment was over, when necessary the doctor creates new EHR records, therefore operations such as EHR update and deletion are not so common in this scenario.

Despite executing performance tests with up to 1000 users, it is worth to mention that the dataset used during those tests was small when compared with real productive scenarios of big health institutions. It is also fair to mention that we performed the tests using a small virtualized server with both organizations. Differently, in a real scenario each organization would use a separate server with much more hardware resources. The authors deployed the blockchain network to a virtualized server, the results collected with Apache JMeter showed an average response time of 336.95 milliseconds when retrieving 100 EHR assets with 800 users in a period of 2 minutes. The average response time showed a net time free of network time, that the application requires retrieving 100 EHRs in a single GET request. The authors believe that it is possible to improve those times, because considering a real use case scenario the end-user may filter by a specific period. Therefore, the system needs to retrieve less data from the ledger, saving both time and hardware resources.

When the CPU consumption reached around $80 \%$ of the total capacity, the average response time increased drastically by 45 times. This happened when performing the tests with 1000 users. Another unwanted behavior observed was when the CPU resources were completely exhausted while testing with 1500 users, the peers went down and the blockchain application stopped working completely. In a real use case scenario, the systems administrators could adopt prevention mechanisms to avoid such undesired situation and they could for sure size the blockchain hardware to supply the real network needs. The study had some limitations regarding the proposed architecture model and the tests.

The authors' first proposal was to collect the EHR data from the different health providers with their own built API. Once the data arrived in the B4Health system, the converter component converted from any data standard into FHIR. However, we did not implement this scenario as mentioned in the beginning of this section and because this work did not cover the internal HIS aspects, the interoperability aspects with other data standards were also not part of this work. Additionally, since all the involved components were in the same virtual server, it was not possible to test the solution's scalability due to this hardware restriction.

\section{CONCLUSION}

In this study, we presented a blockchain architecture model for electronic health records named B4Health. The institutions have been using the EHR to register the patients' information electronically for many years, however this precious data is in many different systems, creating data silos that make the patient care process less efficient. Many institutions implemented these existing HIS systems using different data standards, making it difficult or not possible to exchange information between them. Motivated to overcome such situation, the authors proposed an architecture model for health records based on blockchain. The main outcome was storing part of an electronic medical record in the developed solution, making it possible to have the EHR data available to authorized stakeholders in a unique viewpoint through a decentralized blockchain application.

Since there are many different available blockchain frameworks, the right choice of the platform is a key factor upon the success of any such solution. That was the reason we explored the two major blockchain frameworks available nowadays, Ethereum and Hyperledger Fabric. The model proposed an architecture for patients to have a unique view of their EHR data dispersed in countless systems. However, we still must confirm many other HIS aspects and business requirements not covered here. For example, due to the test limitations regarding dataset size and hardware resources, it is hard to affirm that the proposed model would be able to massively scale to become a national health network.

The authors must submit the prototype through a very robust performance test in the future. We must also cover another key topic in future studies, the interoperability between B4Health and the already existing HIS solutions. As future work, it is important to discover how to embed intelligence into the converter component, so it could convert EHR data stored in a different data standard to HL7 FHIR automatically without losing the data semantics. After going through this exploratory research, it was possible to prove that the blockchain technology has indeed the potential to address the major challenge concerning EHR data scattered in many locations. The authors were able to show using the framework Hyperledger Fabric that by resolving the open 
challenges mentioned above, all the different parties that need to access the same information in the patient care can benefit from the benefit created by blockchain. One can achieve this goal by working in close collaboration with already existing HIS solutions, as they are the main data source needed by any health care blockchain based solution like B4Health.

\section{ACKNOWLEDGEMENT}

The authors would like to thank the Coordenação de Aperfeiçoamento de Pessoal de Nível Superior - Brasil (CAPES) and Brazilian National Council for Scientific and Technological Development (CNPq) for supporting this work.

\section{REFERENCES}

Alonso S. G. et al, 2019. Proposing New Blockchain Challenges in eHealth. Journal of Medical Systems, pp 43-64.

Angraal, S. et al, 2017. Blockchain Technology Applications in Health Care. Circulation: Cardiovascular Quality and Outcomes, Vol. 10, No. 9, pp 253-265.

Azarma M. et al, 2017. Breaking the Healthcare Interoperability Barrier by Empowering and Engaging Actors in the Healthcare System. Procedia Computer Science, Vol. 113, pp 326-333.

Chen L. et al, 2019. Blockchain based searchable encryption for electronic health record sharing. Future Generation Computer Systems, Vol. 95, pp 420-429.

Dubovitskaya, A. et al, 2017. Secure and Trustable Electronic Medical Records Sharing using Blockchain. AMIA Annual Symposium Proceedings, pp 650-659.

Fan K. et al, 2018. Efficient and Secure Medical Data Sharing Via Blockchain. Journal of Medical Systems, Vol. 42, pp 136.

Mertz L, 2018. (Block) Chain Reaction: A Blockchain Revolution Sweeps into Health Care, Offering the Possibility for a Much-Needed Data Solution. IEEE Journals \& Magazines, Vol. 9, no 3, pp 4-7.

Metler, M. 2016. Blockchain technology in healthcare: The revolution starts here. 2016 IEEE 18th International Conference on e-Health Networking, Applications and Services (Healthcom). Munich, Germany, pp. 1-3.

Pons E. et al 2016. Natural Language Processing in Radiology: A Systematic Review. RSNA Radiology, Vol. 279, pp 329-343.

Reisman M. et al 2017. EHRs: The Challenge of Making Electronic Data Usable and Interoperable. Pharmacy and Therapeutics, Vol. 42, no 9, pp 572-575.

Roehrs A. et al 2019. Analyzing the performance of a blockchain-based personal health record implementation. Journal of Biomedical Informatics, Vol. 92.

Roehrs A. et al 2017. Personal Health Records: A Systematic Literature Review. Journal of Medical Internet Research, Vol. 19, e13.

Roehrs A. et al 2017. OmniPHR: A distributed architecture model to integrate personal health records. Journal of Biomedical Informatics, Vol. 71, pp 70-81.

Vuokko R. et al 2017. Results of a systematic literature review from the perspective of secondary use of patient data. International Journal of Medical Informatics, Vol. 97, pp 293-303.

Zhang P. et al 2018. FHIRChain: Applying Blockchain to Securely and Scalably Share Clinical Data. Computational and Structural Biotechnology Journal, Vol. 16, pp 1-23. 